diabetes mellitus (DM), 5 of which were diagnostic at presentation. 67 (45\%) had undiagnosed dyslipidaemia. Of the 63 younger patients who had echocardiograms prior to discharge, $37(48 \%)$ had evidence of left ventricular dysfunction.

Conclusion The gender imbalance observed in STEMI presentations was exaggerated in younger patients. Our study correlates with previous literature suggesting that smoking carries a greater attributable risk for AMI in patients $\leq 45$ years and reinforces the importance of smoking cessation in young males. These patients have high rates of undiagnosed DM and dyslipidaemia. The high rate of LVD is likely to have a significant impact on health outcomes in this young population.

\section{MANAGMENT OF AORTIC ROOT ANEURYSMS; A SINGLE SURGEON EXPERIENCE OVER TWENTY YEARS}

K O' Sullivan, A Graham. Royal Victoria Hospital, Belfast, UK

\subsection{6/heartjn|-2021-ICS.7}

Introduction The aortic root is a complex anatomical structure. For many years, the Bentall operation was considered the gold standard approach to aortic root aneurysms regardless of the presence of absence of intrinsic lesions of the aortic valve. More recently valve sparing root replacement (VSRR) has been developed to avoid the requirement for anticoagulation particularly in younger patient cohorts with structurally normal valves and functional regurgitation. The purpose of this study is to review a single surgeon experience of managing aortic root aneurysms over twenty years. This includes the introduction of valve sparing root replacement and the associated learning curve.

Methods A retrospective institutional review of all patients undergoing aortic root surgery between 2003-2020 was undertaken. Data was retrieved from an institutional database search and patient follow-up attained from up to date regional electronic care records. Graphpad prism was used to carry out Kaplan Meier survival analysis comparing groups. A p value $<0.05$ was considered statistically significant [table 1].

Results A total of 62 aortic root procedures were undertaken, 42 (69\%) Bentall and 20 (31\%) VSRR. VSRR was commenced in 2007 and the frequency increased over time. Of 23 VSRR undertaken, 20 were successful. Three of these

Abstract 7 Table 1 Patient characteristics

\begin{tabular}{llll}
\hline & TRR $\mathrm{n}=42$ & VSRR $\mathrm{n}=20$ & Total $\mathrm{n}=\mathbf{6 2}$ \\
\hline Reoperation for Bleeding & $6(14 \%)$ & $3(15 \%)$ & $9(14.5 \%)$ \\
Other Reoperation - Early & $3(7 \%)$ & $0(0 \%)$ & $3(5 \%)$ \\
Other Reoperation - Late & $1(2 \%)$ & $1(5 \%)$ & $2(3 \%)$ \\
Permanent CVA & $4(\%)$ & $0(0 \%)$ & $4(6 \%)$ \\
PPM & $1(2 \%)$ & $1(5 \%)$ & $2(3 \%)$ \\
Surgical infection - Early and late & $5(12 \%)$ & $0(0 \%)$ & $5(8 \%)$ \\
Prolonged ICU (in elective cases) & $3(7 \%)$ & $0(0 \%)$ & $3(5 \%)$ \\
Prosthetic valve endocarditis & $3(7 \%)$ & $0(0 \%)$ & $3(5 \%)$ \\
Aortic graft infection & $2(5 \%)$ & $0(0 \%)$ & $2(3 \%)$ \\
Lower respiratory tract infection & $0(0 \%)$ & $1(5 \%)$ & $1(2 \%)$ \\
\hline
\end{tabular}

were for acute dissection. Mean follow up for Bentall patients was 98 months, for VSRR 62 months. Complications of Bentall included prosthetic valve endocarditis $n=3(7 \%)$, graft infection $n=2(5 \%)$, late cerebrovascular events $n=3$ (7\%). There was no significant difference in survival between groups.

Conclusion While Bentall remains the gold standard therapy for aortic root aneurysms in many cases, valve sparing root replacement is an excellent option for well selected patients and our series demonstrated a lower incidence of serious endocarditis and graft infections. Furthermore, it carries the added advantage of not requiring anticoagulation in younger patients.

\section{STENT OPTIMIZATION USING OPTICAL COHERENCE TOMOGRAPHY AND ITS PROGNOSTIC IMPLICATIONS AFTER PERCUTANEOUS CORONARY INTERVENTION}

\begin{abstract}
1,2,3 H Rai, ${ }^{1} \mathrm{~F}$ Harzer, ${ }^{4} \mathrm{~T}$ Otsuka, ${ }^{5,6}$ YS Abdelwahed, ${ }^{7} \mathrm{P}$ Antuña, ${ }^{8} \mathrm{~F}$ Blachutzik, ${ }^{9} \mathrm{~T}$ Koppara, ${ }^{4} \mathrm{~L}$ Räber, ${ }^{5} \mathrm{DM}$ Leistner, ${ }^{7} \mathrm{~F}$ Alfonso, ${ }^{8} \mathrm{H}$ Nef, ${ }^{1} \mathrm{M}$ Seguchi, ${ }^{1} \mathrm{~A}$ Aytekin, ${ }^{1} \mathrm{E}$ Xhepa, ${ }^{1} \mathrm{~S}$ Kufner, ${ }^{1} \mathrm{~S}$ Cassese, ${ }^{9} \mathrm{~K}$-L Laugwitz, ${ }^{2,10} \mathrm{RA}$ Byrne, ${ }^{1,11} \mathrm{~A}$ Kastrati, ${ }^{1,11} \mathrm{M}$ Joner. ${ }^{1} \mathrm{~K}$ Kinik für Herz- und Kreislauferkrankungen, Deutsches Herzzentrum München, Germany; ${ }^{2}$ Cardiovascular Research Institute Dublin, Mater Private Dublin, Ireland; ${ }^{3}$ School of Medicine, RCSI University of Medicine and Health Sciences; ${ }^{4}$ Universitätsklinik für Kardiologie, Inselspital Bern, Bern, Switzerland; ${ }^{5}$ Charité - Universitätsmedizin Berlin, Germany; ${ }^{6}$ DZHK (German Centre for Cardiovascular Research), partner site Berlin, Germany: ${ }^{7}$ Hospital Universitario de La Princesa and Universidad Autónoma de Madrid, Spain; ${ }^{8}$ Universitätsklinikum Gießen und Marburg, Giessen, Germany; ${ }^{9}$ Klinikum rechts der Isar, Munich, Germany; ${ }^{10}$ School of Pharmacy and Biomolecular Sciences, RCSI University of Medicine and Health Sciences: ${ }^{11}$ DZHK (German Centre for Cardiovascular Research), partner site Munich Heart Alliance, Munich, Germany
\end{abstract}

\subsection{6/heartin|-2021-ICS.8}

Introduction Stent under-expansion has been known to be associated with worse outcomes. We sought to define optical coherence tomography (OCT) assessed optimal stent expansion index (SEI) which associates with lower incidence of major adverse cardiac events (MACE) during follow-up.

Methods We included 370 lesions who underwent PCI during 2012-2018 at five high volume European centers, with their final procedural results imaged using OCT. Stent expansion index (SEI) was calculated separately for both stent halves by dividing minimum stent area by the mean reference lumen area [(proximal reference area + distal reference area)/2]. (Figure 1, Panel A) Follow-up MACE was defined as a composite of all-cause death, myocardial infarction, stent thrombosis and target lesion revascularization.

Results A total of 316 cases (370 lesions) were analysed. Minimal stent area (MSA) of the whole cohort was 6.02 (4.65, $7.93) \mathrm{mm}^{2}$, while SEI was $0.79(0.71,0.86)$. During a median follow-up duration of $557(326,1,096)$ days, we recoded 47 (12.7\%) incidences of MACE. Receiver operating characteristic (ROC) curve analysis identified 0.85 as SEI cut-off powered to predict $\mathrm{MACE}$ during follow-up $(\mathrm{AUC}=0.60$, sensitivity= 0.85 , specificity $=0.34$ ). (Figure 1, Panel B) MACE was observed in $38 / 249(15.3 \%)$ of lesions with $\mathrm{SEI}<0.85$ and in $9 / 121(7.4 \%)$ of lesions with $\mathrm{SEI} \geq 0.85 \quad(\mathrm{p}=0.03)$. Adaptive Lasso regression identified $\mathrm{SEI}<0.85 \quad(\mathrm{OR}=4.76, \quad 95 \%$ $\mathrm{CI}=1.70-13.30, \mathrm{p}=0.0029)$ and coronary calcification at baseline $\quad(O R=2.70, \quad 95 \% \quad C I=1.11-6.58, \quad p=0.0292) \quad$ as 
A

Stent expansion index (SEI) assessment process
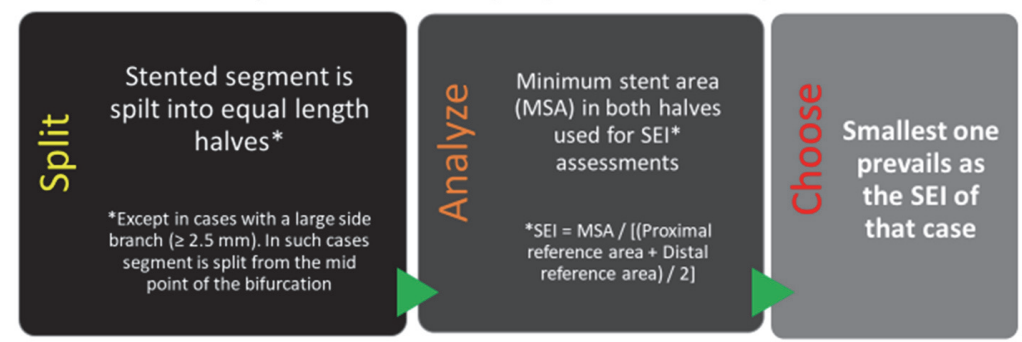

B

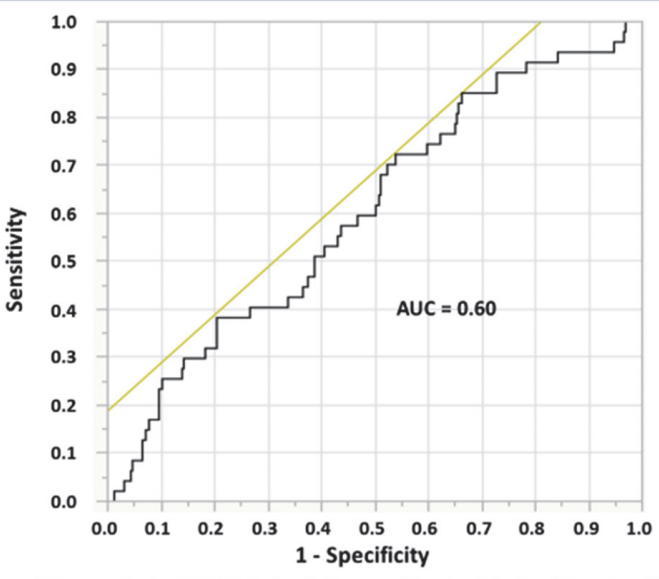

SEI cut-off for predicting MACE during follow-up [Youden's index ( $\mathrm{J}$ ) method] $=\mathbf{0 . 8 5}$ Sensitivity $=0.85$, Specificity $=0.34$

Positive predictive value $=0.16$, Negative predictive value $=0.94$

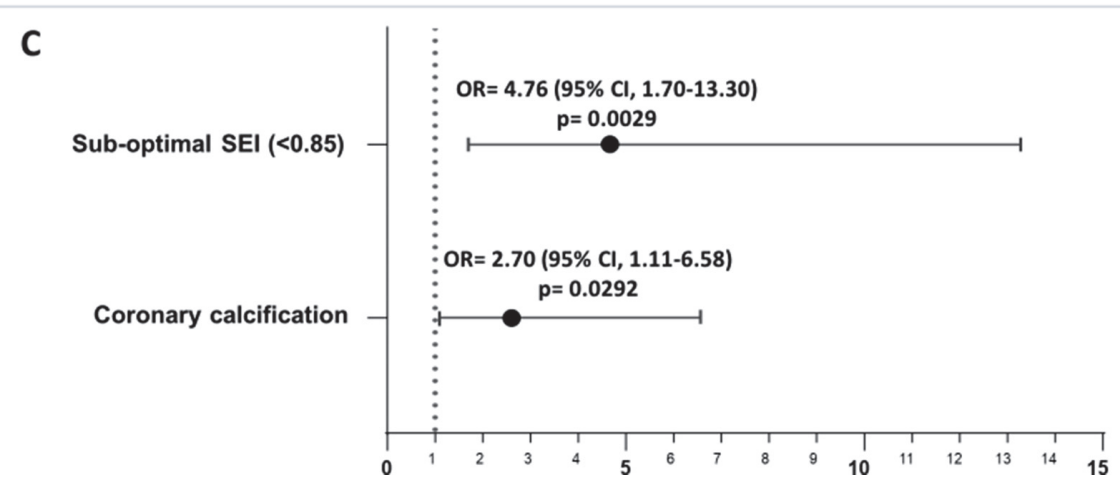

Abstract 8 Figure 1 Stent expansion index (SEI) assessment process

independent predictors of MACE during follow-up. (Figure 1, Panel C).

Conclusions The present study identified $\mathrm{SEI} \geq 0.85$, associated with lower incidence of MACE as optimal cut-off in daily practice. Along with SEI of $<0.85$, coronary calcification was also found to be significantly associated with MACE during post-PCI follow-up.

\section{TARGET VESSEL AND NON-TARGET VESSEL RELATED EVENTS AT 10 YEARS POST PERCUTANEOUS CORONARY INTERVENTION}

${ }^{1} \mathrm{JJ}$ Coughlan, ${ }^{2} \mathrm{~A}$ Aytekin, ${ }^{2} \mathrm{E}$ Xhepa, ${ }^{2} \mathrm{~S}$ Cassese, ${ }^{2} \mathrm{M}$ Joner, ${ }^{2} \mathrm{~T}$ Koch, ${ }^{2} \mathrm{~J}$ Weibe, ${ }^{2} \mathrm{~T}$ Lenz, ${ }^{2} \mathrm{~T}$ Rheude, ${ }^{2} \mathrm{C}$ Pellegrini, ${ }^{2} \mathrm{~S}$ Gewalt, ${ }^{3} \mathrm{~T}$ Ibrahim, ${ }^{3} \mathrm{KL}$ Laugwitz, ${ }^{2} \mathrm{H}$ Schunkert, ${ }^{2} \mathrm{~A}$ Kastrati, ${ }^{2} \mathrm{~S}$ Kufner. 'German Heart Centre Munich, Germany; ${ }^{2}$ Klinik für Herz- und Kreislauferkrankungen, Deutsches Herzzentrum München, Germany; ${ }^{3}$ Klinikum rechts der Isar, Munich, Germany

10.1136/heartjnl-2021-ICS.9
Background Patients who undergo percutaneous coronary intervention (PCI) are at risk of events related to the stented vessel (TVRE) and events related to progression of disease in non-stented vessels (NTVRE). Long-term follow up may be useful to define the relative contribution of TVRE and NTVRE to overall event rates post PCI and to guide patient care. AIM To define the incidence of TVRE and NTVRE through 10 year follow up in patients post PCI with newer generation DES.

Methods The current study is an analysis of patient level data from the ISAR-TEST 4 and ISAR-TEST 5 trials. The endpoints of interest were TVRE (a composite of first target vessel myocardial infarction or target vessel revascularization) and NTVRE (a composite of first non-target vessel MI or non-target vessel revascularization) through 10 years.

Results We included 4,953 patients in this analysis. Through 10 year follow up post PCI, TVRE occurred in 1,238 of 4,953 patients (cumulative incidence: 25.8\%) and NTVRE occurred in 1,442 of 4,953 patients (cumulative incidence: $30.3 \%)$. The majority of events in both groups were 